\title{
A model reference adaptive variable impedance control method for robot
}

\author{
Xinchao Sun ${ }^{1,2}$, Lianyu Zhao ${ }^{1,2}$, and Zhenzhong Liu ${ }^{1,2 .}$ * \\ ${ }^{1}$ Tianjin Key Laboratory for Advanced Mechatronic System Design and Intelligent Control, School of \\ Mechanical Engineering, Tianjin University of Technology \\ ${ }^{2}$ National Demonstration Center for Experimental Mechanical and Electrical Engineering Education, \\ School of Mechanical Engineering, Tianjin University of Technology
}

\begin{abstract}
As a simple and effective force tracking control method, impedance control is widely used in robot contact operations. The internal control parameters of traditional impedance control are constant and cannot be corrected in real time, which will lead to instability of control system or large force tracking error. Therefore, it is difficult to be applied to the occasions requiring higher force accuracy, such as robotic medical surgery, robotic space operation and so on. To solve this problem, this paper proposes a model reference adaptive variable impedance control method, which can realize force tracking control by adjusting internal impedance control parameters in real time and generating a reference trajectory at the same time. The simulation experiment proves that compared with the traditional impedance control method, this method has faster force tracking speed and smaller force tracking error. It is a better force tracking control method.
\end{abstract}

\section{Introduction}

With the progress of robotics technology, robots are widely used in contact operations such as grinding, assembly, space operations, medical surgeries and so on. Contact operation requires the robot to meet both position accuracy and force accuracy. The introduction of impedance control makes this a reality. The traditional impedance control method adopts fixed parameter control, it is difficult to realize effective force tracking control, and it is easy to produce larger force tracking error. Therefore, many scholars have improved this method. Literature [2,3] uses the learning impedance control method to achieve effective force tracking control, but the training process of this method requires a lot of data and is difficult to apply to real-time systems. Literature $[4,5]$ adopt fuzzy control to realize force tracking control of vertebral lamina milling. Although fuzzy control has strong robustness and fault tolerance, simple fuzzy processing of information will lead to reduction of control accuracy and deterioration of dynamic quality of the system. Literature [6,7] adopt neural network control method to achieve force tracking control, but this method is usually designed complex and it takes several weeks or even longer to training data. Adaptive control is widely used because it does not require sample training, and can adjust impedance control parameters in real time according to force errors. Literature [8] proposed an adaptive impedance controller

* Corresponding author: zliu@email.tjut.edu.cn 
based on the Hammerstein model. Experiments on the bionic wrist joint proved that this method can still effectively limit the contact force when the environment changes. Literature [9] proposed a nonlinear bilateral adaptive impedance control method for teleoperation systems. Literature [10] proposed a novel backstepping adaptive impedance control for an upper limb rehabilitation robot.

This paper combines the advantages of variable impedance control and adaptive control, and proposes a new impedance control method-model reference adaptive variable impedance control. In the rest of the article, three impedance control methods are introduced and simulated, which are traditional position-based impedance control method, model reference adaptive impedance control method and model reference adaptive variable impedance control method.

\section{Impedance control methods}

\subsection{Traditional position-based impedance control (TPIC)}

The system structure diagram of TPIC is shown in Fig. 1 . Where, $p_{d}$ and $p_{r}$ respectively represent the desired position and reference position of the end of the manipulator; $p_{m}$ is the actual measured position of the end of the manipulator; $p_{e}$ is the imaginary contact position; $e_{f}$ is the difference between the desired contact force $f_{d}$ and the actual measured contact force $f_{m} ; k_{e}$ is the environmental stiffness and $f_{m}=k_{e}\left(p_{m}-p_{e}\right)$.

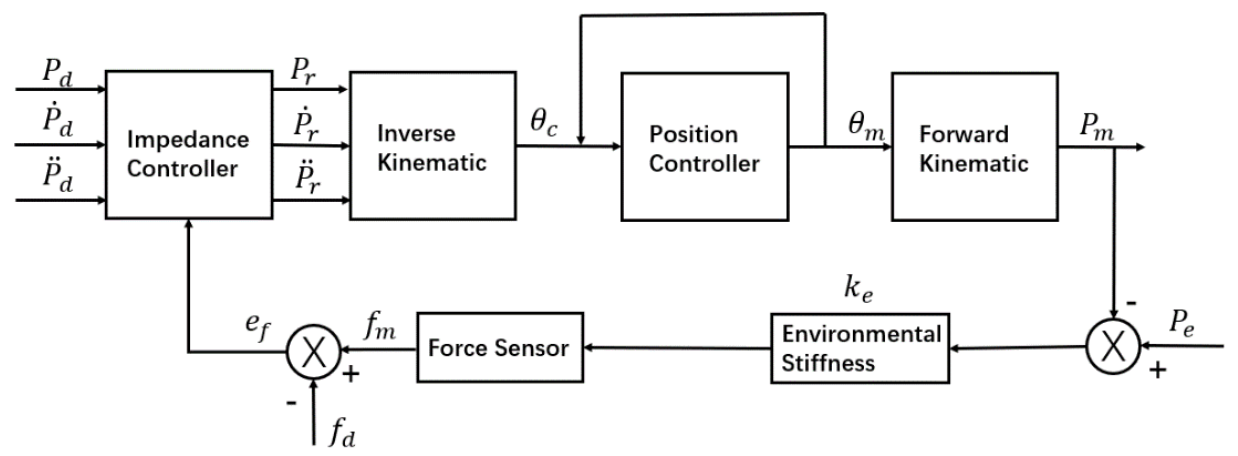

Fig. 1. The system structure diagram of TPIC.

The mathematical model of impedance control [1] is as follows:

$$
m_{d}\left(\ddot{p_{d}}-\ddot{p_{r}}\right)+b_{d}\left(\dot{p}_{d}-\dot{p}_{r}\right)+k_{d}\left(p_{d}-p_{r}\right)=e_{f}
$$

where, $m_{d}, b_{d}$ and $k_{d}$ represent the inertia parameter, damping parameter and stiffness parameter of impedance control respectively.

\subsection{Model reference adaptive impedance control (MRAIC)}

The internal parameters of TPIC cannot be automatically adjusted according to environmental changes. When the environment changes greatly, the control system may be unstable or produce large force tracking error. MRAIC [11] can correct the trajectory in real time, and it can keep the system stable even when the external environment changes greatly. The system structure diagram of MRAIC is shown in Fig. 2. 


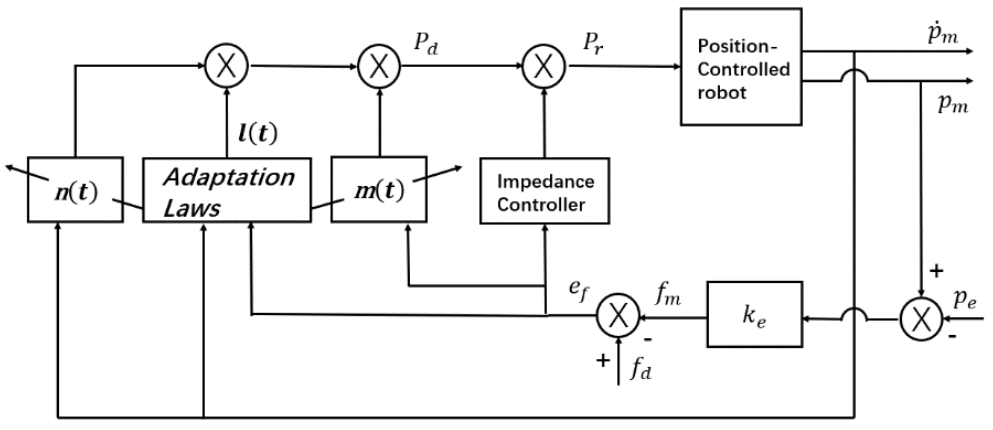

Fig. 2. The system structure diagram of MRAIC.

In this section, the adaptive control law defined in [11] is utilized as follows:

$$
\begin{gathered}
p_{d}(t)=l(t)+m(t) e_{f}(t)-n(t) \dot{p}_{m}(t) \\
l(t)=l(0)+\alpha \int_{1}^{t} h(t) d t+\alpha_{2} h(t)-\sigma_{1} \int_{0}^{t} l(t) d t \\
m(t)=m(0)+\beta_{1} \int_{0}^{t} h(t) e_{f}(t) d t+\beta_{2} h(t) e_{f}(t)-\sigma_{2} \int_{0}^{t} m(t) d t \\
n(t)=n(0)-\lambda_{1} \int_{0}^{t} h(t) \dot{p_{m}}(t) d t-\lambda_{2} h(t) \dot{q}_{m}(t)-\sigma_{3} \int_{0}^{t} n(t) d t \\
h(t)=\gamma_{1} e_{f}(t)+\gamma_{2} \dot{p}_{m}(t)
\end{gathered}
$$

Among them, $\alpha_{1}, \alpha_{2}, \beta_{1}, \beta_{2}, \gamma_{1}, \gamma_{2}, \lambda_{1}, \lambda_{2}, \sigma_{1}, \sigma_{2}$ and $\sigma_{3}$ are small positive numbers to ensure the robustness of the system.

\subsection{Model reference adaptive variable impedance control (MRAVIC)}

MRAIC is a control method that generates trajectory corrections through force errors, but it cannot change the internal parameters of the impedance controller. Therefore, we introduce the idea of variable impedance control into MRAIC, and propose a new impedance control method-MRAVIC. The system structure diagram of MRAVIC is shown in Fig. 3.

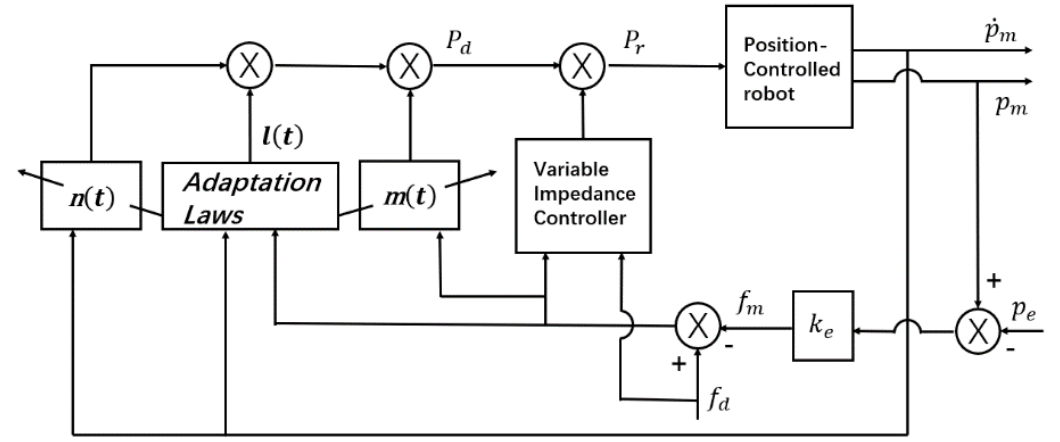

Fig. 3. The system structure diagram of MRAVIC.

When the variable impedance control is adopted, the internal parameters are changed. The Eq. (1) can be rewritten as: 


$$
m(t) \ddot{e_{p}}+b(t) \dot{e}_{p}+k(t) e_{p}=e_{f}
$$

Where,

$$
k(t)=\left(k_{p} e_{f}+k_{q} \dot{e}_{f}\right) / e_{p}
$$

Among them, $k_{p}$ and $k_{q}$ are the scale factor and differential factor of force error, respectively. Substituting Eq. (8) into Eq. (7):

$$
\begin{aligned}
0 & =m(t) \ddot{e}_{p}+b(t) \dot{e}_{p}+k_{p} e_{f}+k_{q} \dot{e}_{f}-e_{f} \\
& =m(t) \ddot{e}_{p}+b(t) \dot{e}_{p}+k_{p}^{\prime} e_{f}+k_{q} \dot{e}_{f}
\end{aligned}
$$

Where, $k_{p}^{\prime}=k_{p}-1$.

When Eq. (9) is stable, $\ddot{e}_{p}=0, \dot{e}_{p}=0, \dot{e}_{f}=0$, at this time, $k_{p}^{\prime} e_{f}=0$, in other words, $e_{f \rightarrow \text { stable }}=0$. Therefore, the parameter control law represented by Eq. (8) can ensure the stability of the system in the contact state.

The impedance control with variable parameters does not satisfy the passivity condition, so its stability should be analysed. Take the scalar Lyapunov candidate function as follows:

$$
V=\frac{1}{2} m \dot{p}^{2}+\frac{1}{2} k_{m} e_{f}^{2}
$$

The form of $k_{m}$ will be explained later. Taking the derivative of Eq. (10) to get Eq. (11).

$$
\dot{V}=\frac{1}{2} \dot{m} \dot{p}^{2}+m \ddot{p} \dot{p}+k_{m} e_{f} \dot{e}_{f}
$$

And then

$$
\dot{V}=\left(\frac{1}{2} \dot{m}-b+k_{q} k_{e}\right) \dot{p}^{2}+\left(k_{p}^{\prime}-k_{m} k_{e}\right) e_{f} \dot{p}
$$

If $k_{m}$ is defined as $k_{m}=k_{p}^{\prime} / k_{e}$, the second half of Eq. (12) can be eliminated. Eq. (12) can be rewritten as

$$
\dot{V}=\left(\frac{1}{2} \dot{m}-b+k_{q} k_{e}\right) \dot{p}^{2} \leq 0
$$

If Eq. (13) is to be established, that is, $\dot{V}$ is semi-negative definite, as long as Eq. (14) is satisfied.

$$
\frac{1}{2} \dot{m}-b+k_{q} k_{e} \leq 0
$$

Therefore, the proof of the stability of Eq. (7) is obtained.

\section{Simulation}

Using Matlab/Simulink to simulate the three impedance control methods. The object of the simulation experiment is a JAKA Zu7 manipulator with a force sensor and a bone drill at the end. The three-dimensional model of the robot is shown in Fig. 4. The simulation experiment simulates the robot drawing a circle on a plane. The expected motion trajectory of the bone drill on the xy plane is a circle with a radius of $0.03 \mathrm{~m}$, and the position of the centre of the circle in the xy coordinate system is $(-0.4705,-0.112)$. When the bone drill is in contact with the contact surface, the bone drill is perpendicular to the contact surface, and the contact force is controlled in the z-axis direction. The desired contact force is set to $25 \mathrm{~N}$. When the force 
tracking error is less than $0.15 \mathrm{~N}$, the bone drill is considered to have been in contact with the plane.

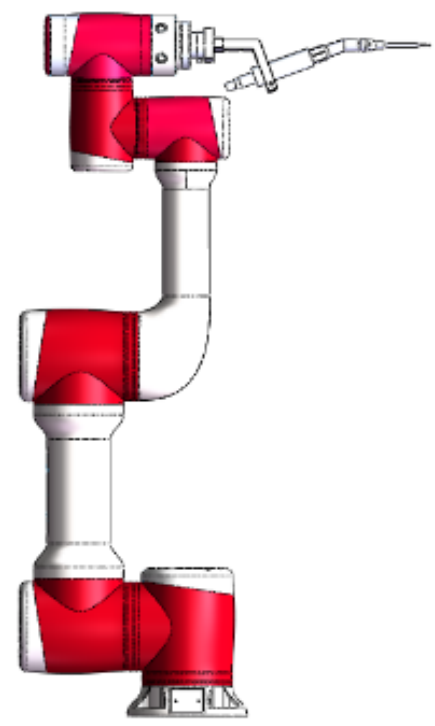

Fig. 4. 3D model of the robot.

\subsection{The simulation of TPIC}

The simulation diagram of TPIC is shown in Fig. 5. The hypothetical contact position $p_{e}$ is set to $0.853 \mathrm{~m}$, the environmental stiffness $k_{e}$ is set to $1000 \mathrm{~N} / \mathrm{m}$ and the simulation time is set to $10 \mathrm{~s}$. After repeated debugging, when $m_{d}$ is set to $0.5 \mathrm{~kg}, b_{d}$ is set to $800 \mathrm{Ns} / \mathrm{m}$ and $k_{d}$ is set to $2 \mathrm{~N} / \mathrm{m}$, it has a good control effect.

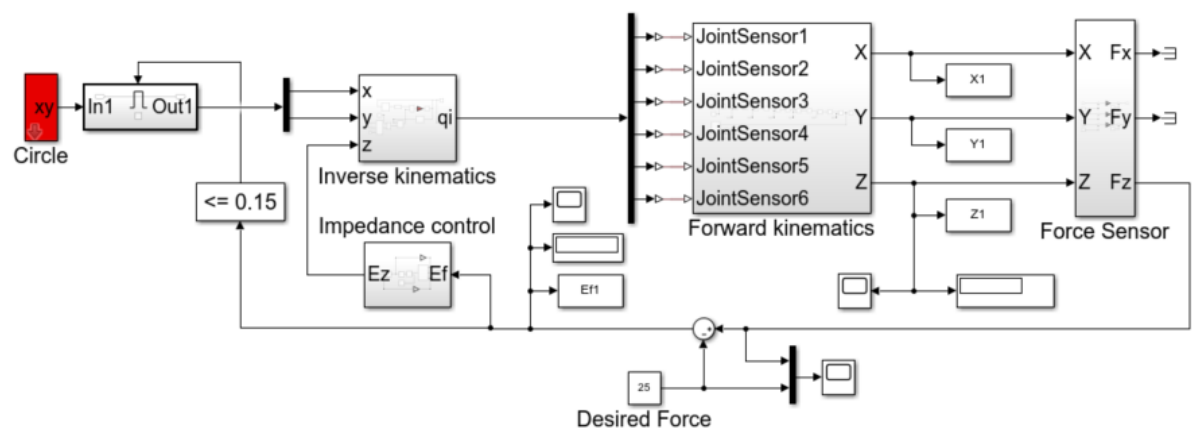

Fig. 5. The simulation diagram of TPIC.

The simulation result is shown in Fig. 6. The simulation result shows that the system starts to enter the steady state after $2.850 \mathrm{~s}$, and the average value of the absolute value of the force tracking errors after entering the steady state is $0.056 \mathrm{~N}$. After the steady state, the z-axis position of the bone drill is stable at $0.878 \mathrm{~m}$, and the trajectory of the xy plane of the bone drill is the desired circular trajectory. 


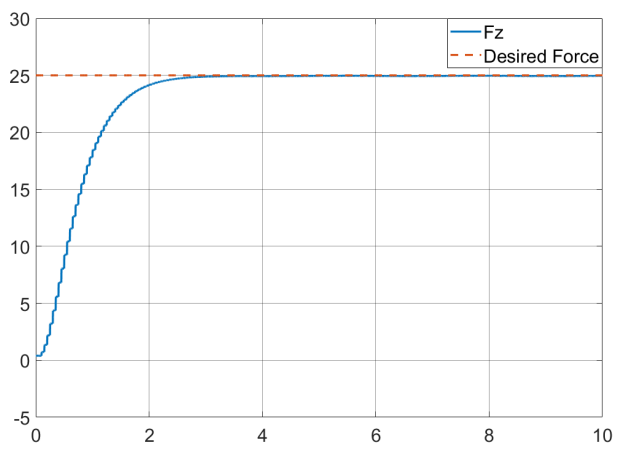

(a) The effect of force tracking.

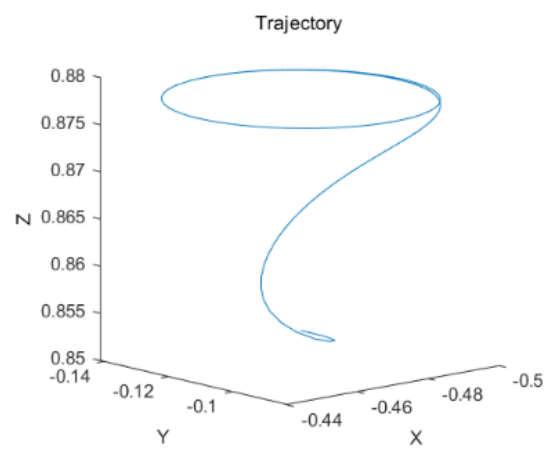

(b) The trajectory of bone drill.

Fig. 6. Simulation result.

\subsection{The simulation of MRAIC}

Taking the adaptive control parameters $\alpha_{1}=\alpha_{2}=0.001, \beta_{1}=\beta_{2}=0.001, \gamma_{1}=\gamma_{2}=$ $0.001, \lambda_{1}=\lambda_{2}=0.001$, the correction coefficients $\sigma_{1}=\sigma_{2}=\sigma_{3}=0.001$ and $l(0)=$ $m(0)=n(0)=0$. The settings of other parameters of the simulation experiment are the same as those of TPIC simulation experiment. The simulation experiment diagram of MRAIC is shown in Fig. 7.

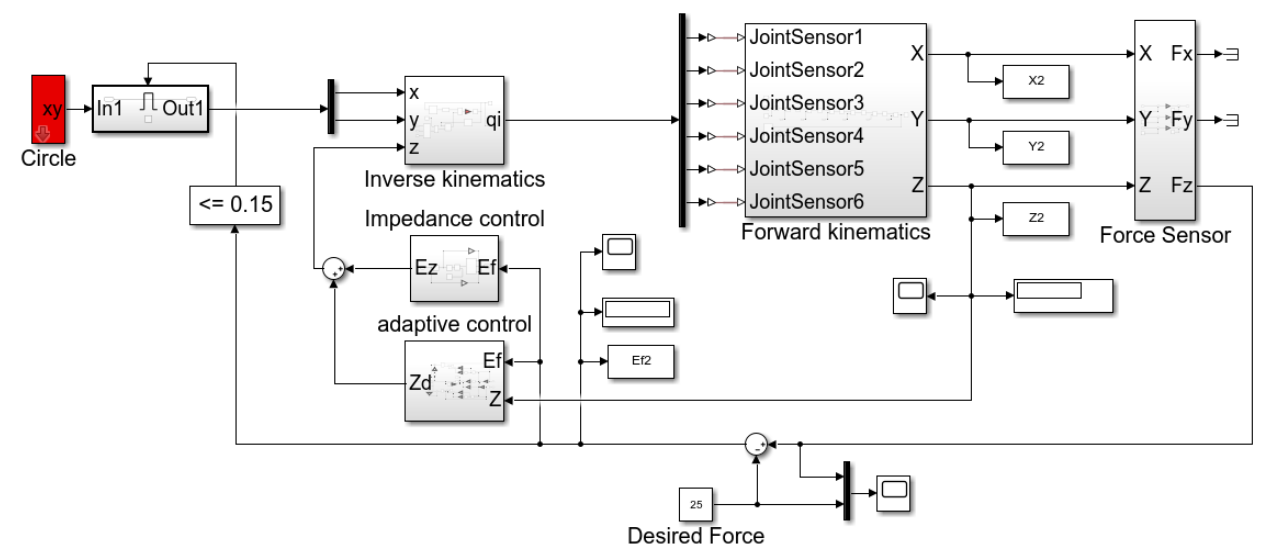

Fig. 7. The simulation experiment diagram of MRAIC.

The simulation result is shown in Fig. 8. The simulation result shows that the system starts to enter the steady state after $2.452 \mathrm{~s}$, and the average value of the absolute value of the force tracking errors after entering the steady state is $0.051 \mathrm{~N}$. After the steady state, the z-axis position of the bone drill is stable at $0.878 \mathrm{~m}$, and the trajectory of the xy plane of the bone drill is the desired circular trajectory. 


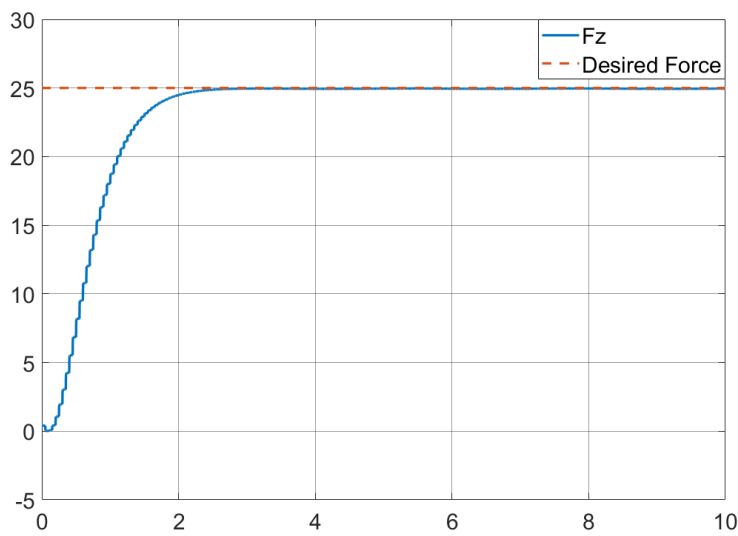

(a) The effect of force tracking.

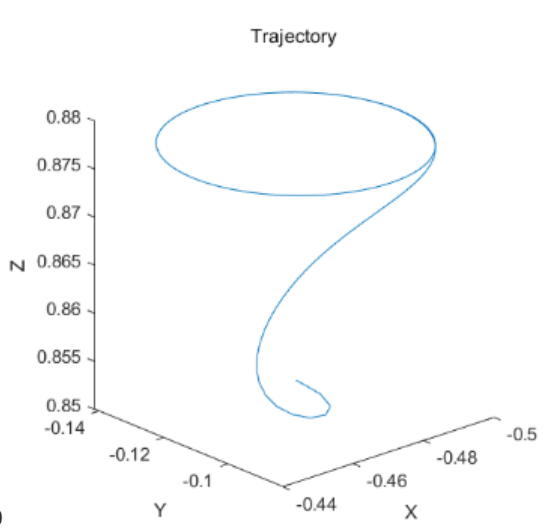

(b) The trajectory of bone drill.

Fig. 8. Simulation result.

\subsection{The simulation of MRAVIC}

The simulation experiment diagram of MRAVIC is shown in Fig. 9. Taking $k_{p}=0.0001$ and $k_{q}=0.0001$. The settings of other parameters are the same as those of MRAIC.

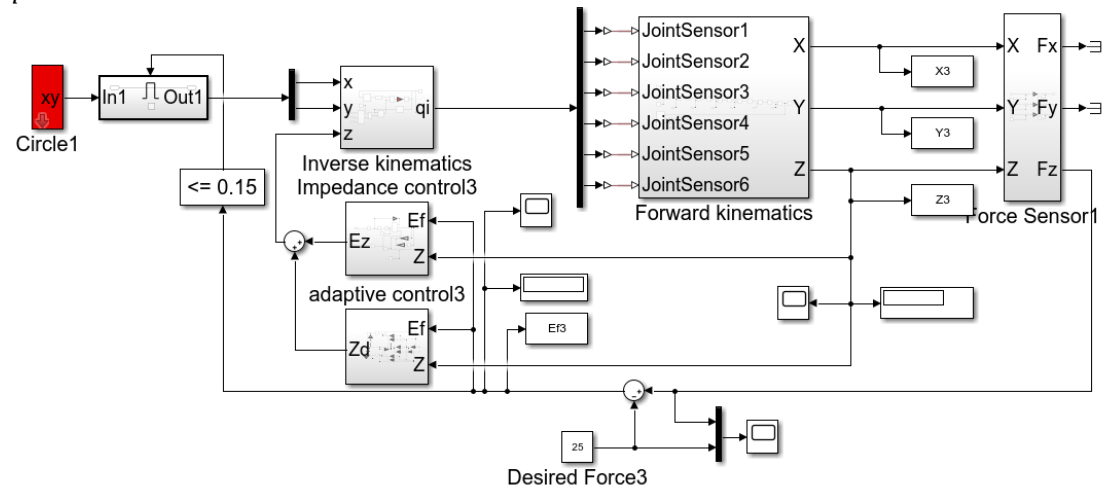

Fig. 9. The simulation experiment diagram of MRAVIC.

The simulation result is shown in Fig. 10. The simulation result shows that the system starts to enter the steady state after $1.952 \mathrm{~s}$, and the average value of the absolute value of the force tracking errors after entering the steady state is $0.011 \mathrm{~N}$. After the steady state, the $\mathrm{z}$ axis position of the bone drill is stable at $0.878 \mathrm{~m}$, and the trajectory of the xy plane of the bone drill is the desired circular trajectory. 


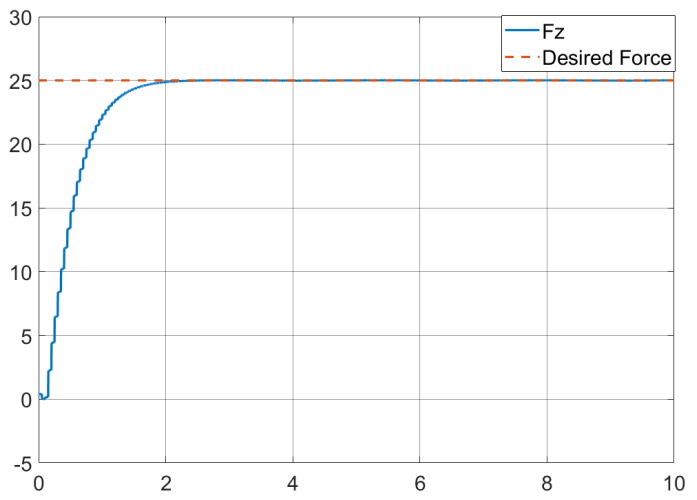

(a) The effect of force tracking.

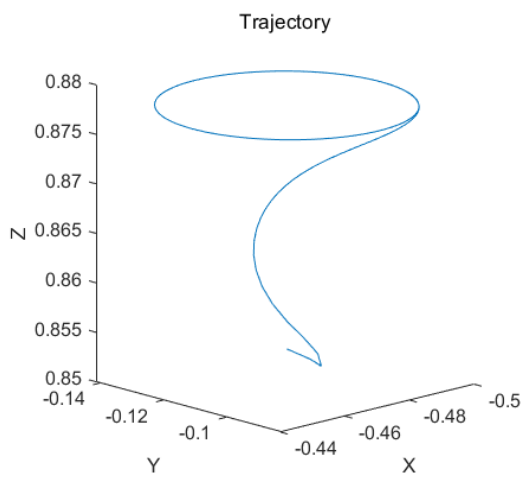

(b) The trajectory of bone drill.

Fig. 10. Simulation result.

\subsection{Analysis of experimental results}

It can be seen from Table 1 that compared with TPIC, MRAIC can effectively improve the force tracking speed, but it cannot effectively reduce the force tracking error. Compared with TPIC, MRAVIC can not only effectively improves the force tracking speed, but also reduces the force tracking error by nearly $80 \%$. Therefore, MRAVIC is a better force tracking control method, which can be used for robot contact operations that require higher force accuracy.

Table 1. Simulation results.

\begin{tabular}{|c|c|c|}
\hline Control method & Time taken to reach steady state/s & $\begin{array}{c}\text { Average value of absolute value of } \\
\text { force tracking error after steady } \\
\text { state } / \mathbf{N}\end{array}$ \\
\hline TPIC & 2.850 & 0.056 \\
\hline MRAIC & 2.452 & 0.051 \\
\hline MRAVIC & 1.952 & 0.011 \\
\hline
\end{tabular}

\section{Conclusion}

In this paper, a model reference adaptive variable impedance control method for robot is designed. This method can realize force tracking control by adjusting internal impedance control parameters and trajectory correction in real time. The simulation results prove that compared with TPIC, MRAVIC has the advantages of faster force tracking speed and smaller force tracking error and it is a better impedance control method. This research provides theoretical support for the application of robots in medical operations, space operations and other occasions that require higher force accuracy.

This study was supported by the National Natural Science Foundation of China (Grant No. 61873188).

\section{References}

1. N. Hogan, Imped. Contr., J. Dyn. Syst. Meas. Contr., 107 (1985). 
2. C. Li, Z. Zhang, G. Xia, et al., Eff. Force Contr. Learn. System Ind. Robots Based Var. Imped. Contr., Sensors, 18 (2018).

3. X. Li, Y. Liu, H. Yu, Iter. Learn. Imped. Contr. rehab. robots driv. series elastic act., Autom., 90(2018)

4. Z. Deng, H. Jin, Y. Hu, et al., Fuzzy force contr. state det. Vert. lamina mill., Mech., 35 (2016).

5. Fan L., Gao P., Zhao B., et al., Safety Contr. Strat. Vert. Lamina Mill. Task, CAAI Trans. Intell. Techn., 1(2016)

6. X. Chen, N. Wang, H. Cheng, et al., Neural Learn. Enh. Var. Adm. Contr. for HumanRobot Coll., IEEE Access, 8(2020)

7. H. Choi, S. Jung, Tel. Contr. Posit. Based Imped. Force Contr. Mobile Robot Neural Network Learn. Exp. Studies, Asian J. Contr., 22 (2020)

8. H. Yang, X. Gao, Y. Chen, et al., Hamm. Adapt. Imped. Contr. Bionic Wrist Joint Act. Pneum. Muscles, IEEE Access, 7(2019)

9. M. Sharifi, S. Behzadipour, H. Salarieh, Nonl. Bilat. Adapt. Imped. Cont. Appl. Tel. Tel., ASME. J. Dyn. Sys. Meas. Contr., 138(2016)

10. J. Bai, A. Song, T. Wang, et al., novel backst. Adapt. Imped. Contr. upper limb reh. robot, Comput. Electr. Engin., 80(2019)

11. H. Seraji, R. Colbaugh, Force track. Imped. Cont., Proc. IEEE Int. Conf. on Robotics Autom., 2(1993) 